\title{
From lesions to cognitive theory
}

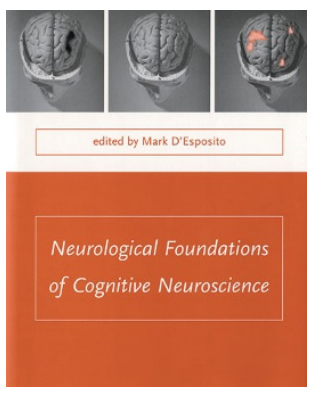

\section{Neurological Foundations of Cognitive Neuroscience}

Cognitive neuroscience has many intellectual roots. The experimental side includes the very different methods of systems neuroscience, human experimental psychology and, of course, functional imaging. The theoretical side has contrasting approaches from neural network theorizing (connectionism), symbolic artificial intelligence, theoretical linguistics and information-processing psychology. Only one area, however, has tightly linked clinical with non-clinical studies and experiments with theoretical modeling. This is neuropsychology, the study of cognitive disabilities of brain-damaged patients-the subject of this book.

Neuropsychological methods lack the raw power of functional imaging methods such as fMRI. Thus group studies in neuropsychology have much more limited anatomical precision than imaging studies. Moreover, detailed theoretical investigations almost entirely depend on the availability of a few highly similar patients. Nevertheless, the logic of extrapolation from experimental findings to cognitive theory is much more transparent and better worked out than for functional imaging in general. Moreover, functional imaging studies, with the striking exception of those using the Price/Friston conjunction designs, tend for economic reasons to be based on one or two experiments. A typical good neuropsychological case study can involve 10 to 15 experiments, allowing much tighter theoretical interpretations.

This book aims to bridge between the anatomically more powerful empirical methods of cognitive neuroscience and the complementary strengths of neuropsychology. The result is a very valuable vol-

Tim Shallice is at the Institute of Cognitive Neuroscience, University College London, 17 Queen Square, London WC1N 3AR, UK, and the International School for Advanced Studies (SISSA), Trieste, Italy. ume. The editor, Mark D'Esposito, has assembled a marvellous set of authors - the cream of the behavioral neurologists of his generation in the English-speaking world. The volume is sharply differentiated from the normal run of edited collections, as each chapter is on a different clinical syndrome. Yet all have a common plan, to which the authors have held very well. Each chapter begins with a case history to make the material concrete. Next is a brief historical perspective and a wider clinical survey of related disorders, followed by a discussion of the relationship of the disorders to processing theories of the cognitive domain. Each chapter ends with correspondences to functional imaging results, and, where relevant, systems neuroscience findings.

The chapters are all of a high standard. Some are exceptionally good-such as those of Hodges on semantic dementia, Coslett on the acquired dyslexias, Gitelman on acalculia (a deficit in arithmetic operations), Alexander on transcortical motor aphasia and Binder on Wernicke's aphasia. They each exhibit a command of many different disciplines and give clear accounts of theoretical positions. In a mere 25 pages or so, they review the link to the other relevant approaches in a disciplined, detailed, definitive and very readable way.

Given the complexity of their brief chapters, the emphasis of individual authors naturally varies. Hodges shows implicitly what some other authors do not, that the modern quantitative neuropsychological case study is far from the loose verbal descriptions typical of early clinical neurology, and uses it in a masterly fashion to address various theoretical issues - even if I disagree with some of his conclusions. Alexander and also Aguirre, on topographical disorientation, differentiate related disorders and link them to anatomy in a convincing way. Coslett, Binder and also Rafal on Balint's syndrome show most effectively how patient evidence can be used to relate to models of normal function.
Mega, on amnesia, makes telling links to the systems neuroscience evidence.

Yet something important is missing. The editor, having selected splendid authors, given them an excellent brief, and kept them to it, then seems to have assumed the job was done. However, the chapters reflect the theoretical confusion in cognitive neuroscience, a hotchpotch of methods and theories that relate to each other in a poorly thought-out way. Some authors use information processing models; others use connectionist ones. Many use the vague verbal characterization of processes that unfortunately passes for theorizing in many empirical studies. Thus, 'working memory' is used as an uncontentious explanatory term, even though Miyake and Shah, in their excellent review, showed that the term has almost as many referents as theoreticians. Moreover, there are no well-worked procedures for relating the empirical evidence of cognitive neuroscience to theories, with the exception of those derived from cognitive neuropsychology - and even this clarity is accompanied by major disputes about methods. Some, such as Caramazza and Coltheart, think that extrapolation to normal function is only possible when evidence from single patients is used; averaging across them leads to artifact. Probably still the more common view is that such singlecase studies remain unscientific and that valid evidence for extrapolation to normal function is derived only from the average results of groups of patients related by anatomy or disorder.

The authors differ in their favored methods, but there is no hint of the general methodological and conceptual problems that beset the field and indeed cognitive neuroscience in general. The brevity of the chapters necessitated short-cuts and glossing over problems of method. However, to make the book exceptional, a proper overview chapter reviewing the conceptual and methodological problems of the area and situating the chapters was essential. The preface is a mere single page that does not mention such issues. It is also unclear why certain syndromes were selected, and other apparently equally suitable ones—such as object and spatial agnosia, Broca's and conduction aphasia, orbital frontal disorders, and agraphia (inability to write) were ignored.

The reader already knowledgeable on both sides of the basic science/clinical divide will find this volume of great value. A reader with less clinical expertise would be well advised to read it in conjunction with a modern neuropsychology text such as that of Andrewes. 